\title{
Non-active implantable device treating acid reflux with a new dynamic treatment approach: 1-year results
}

\section{RefluxStop ${ }^{\mathrm{TM}}$ device; a new method in acid reflux surgery obtaining CE mark}

Miloš Bjelović ${ }^{*}$, László Harsányi ${ }^{2}$, Áron Altorjay ${ }^{3}$, Zsolt Kincses ${ }^{4}$, Peter Forsell ${ }^{5}$ and Investigators of the RefluxStop ${ }^{\text {TM }}$ Clinical Investigation Study Group

\begin{abstract}
Background: RefluxStop ${ }^{\mathrm{TM}}$ is an implantable, non-active, single use device used in the laparoscopic treatment of GERD. RefluxStop ${ }^{\mathrm{TM}}$ aims to block the movement of the LES up into the thorax and keep the angle of His in its original, anatomically correct position. This new device restores normal anatomy, leaving the food passageway unaffected.

Methods: In a prospective, single arm, multicentric clinical investigation analyzing safety and effectiveness of the RefluxStop ${ }^{\text {TM }}$ device to treat GERD, 50 subjects with chronic GERD were operated using a standardized surgical technique between December 2016 and September 2017. They were followed up for 1 year (CE-mark investigation 6months). Primary safety outcome was prevalence of serious adverse events related to the device, and primary effectiveness outcome reduction of GERD symptoms based on GERD-HRQL score. Secondary outcomes were prevalence of adverse events other than serious adverse events, reduction of total acid exposure time in 24-h pH monitoring, and reduction in average daily PPI usage and subject satisfaction.

Results: There were no serious adverse events related to the device. Average GERD-HRQL total score at 1 year improved $86 \%$ from baseline $(p<0.001)$. 24-h pH monitoring compared to baseline showed a mean reduction percentage of overall time with $\mathrm{pH}<4$ from 16.35 to $0.80 \%$ at the 6 -month visit $(p<0.001)$, with $98 \%$ of subjects showing normal 24-h pH. At 1 year: No new cases of dysphagia were recorded, present in 2 subjects, which existed already at baseline. Regular daily PPI usage occurred in all 50 subjects at baseline. At 1 -year follow-up, only 1 subject took regular daily PPIs due to a too low placement of the device thereby prohibiting its function. None or minimal occasional episodes of regurgitation occurred in $97.8 \%$ of evaluable subjects. Gas bloating disappeared in 30 subjects and improved in 7 subjects.

(Continued on next page)
\end{abstract}

\footnotetext{
*Correspondence: drbjelovic@gmail.com

'Department for Minimally Invasive Upper Digestive Surgery, University Hospital for Digestive Surgery - First Surgical Hospital, Clinical Center of Serbia; University of Belgrade, School of Medicine, Belgrade, Serbia Full list of author information is available at the end of the article
}

(c) The Author(s). 2020 Open Access This article is licensed under a Creative Commons Attribution 4.0 International License, which permits use, sharing, adaptation, distribution and reproduction in any medium or format, as long as you give appropriate credit to the original author(s) and the source, provide a link to the Creative Commons licence, and indicate if changes were made. The images or other third party material in this article are included in the article's Creative Commons licence, unless indicated otherwise in a credit line to the material. If material is not included in the article's Creative Commons licence and your intended use is not permitted by statutory regulation or exceeds the permitted use, you will need to obtain permission directly from the copyright holder. To view a copy of this licence, visit http://creativecommons.org/licenses/by/4.0/ The Creative Commons Public Domain Dedication waiver (http://creativecommons.org/publicdomain/zero/1.0/) applies to the data made available in this article, unless otherwise stated in a credit line to the data. 
(Continued from previous page)

Conclusion: The new principle of RefluxStop ${ }^{\text {TM }}$ is safe and effective to treat GERD according to investigation results. At 1-year follow-up, both the GERD-HRQL score and 24-h pH monitoring results indicate success for the new treatment principle. In addition, with the dynamic treatment for acid reflux, which avoids compressing the food passageway, prevalence of dysphagia and gas bloating are significantly reduced.

Trial registration: ClinicalTrials.gov, NCT02759094. Registered 3 May, 2016,

Keywords: Gastroesophageal reflux disease (GERD), Treatment, Surgery, RefluxStop ${ }^{\mathrm{TM}}$, Dysphagia, pH monitoring, GERD$\mathrm{HRQL}$, Gas bloating

\section{Background}

Anti-reflux surgery has been focused on supporting the Lower Esophageal Sphincter (LES) for the past 65 years. Nissen fundoplication [1-22], as one standard of care, compresses the food passageway causing dysphagia, odynophagia and inability to belch or vomit as well as associated gas bloating. The long-term result is also plagued by a $36 \%$ failure rate when including $25 \%$ PPI users and an $11 \%$ reoperation rate [23]. This is likely caused by the fact that the cuff becomes thinner and more inelastic over time due to inactivation of the stomach wall.

RefluxStop $^{\mathrm{Tm}}$ is an implantable, non-active, single use sterile device to be used in the laparoscopic treatment of GERD (Fig. 1).

The thesis behind RefluxStop ${ }^{\mathrm{TM}}$ is that acid reflux is caused by two malfunctioning events, both of which are addressed with this new device. First, the belching process with fundus contraction and simultaneous relaxation of the LES also includes fluid due to the anatomical misalignment of the angle of His. Furthermore, acid reflux is caused by the lower esophageal sphincter temporarily or permanently entering into the chest. The pressure in the abdomen supports the LES to close while when in the chest, due to the abnormal thorax position with weaker pressure support combined with the breathing process, the closing function is often not working properly resulting in acid reflux. During the breathing process, the diaphragm moves up and down. This fact has been underestimated as the cause of acid reflux and a new dynamic anti reflux treatment is needed.

RefluxStop $^{\mathrm{TM}}$ reinforces the fundus to interact with the diaphragm for a dynamic treatment of acid reflux. It is placed on the outside of the stomach top fundus wall with laparoscopic surgery. The RefluxStop ${ }^{\mathrm{Tm}}$ procedure reconstructs the angle of His and reinforces the top part of the stomach (fundus) by invagination of the device in the pocket created out of the anterior wall of the fundus. RefluxStop $^{\text {Tm }}$ aims to block the movement of the LES up into the thorax and keep the angle of His in its original anatomically correct position. This new device restores normal anatomy (with the LES remaining in the abdomen) by dynamically acting like a mechanical stop against the diaphragm muscle parallel to the LES and the hiatus opening in the diaphragm, leaving the food passageway unaffected. Therefore, side effects associated with "gold standard" surgery are reduced when avoiding compression of the food passageway.

\section{Methods}

\section{Study design and objectives}

In a prospective, single arm, multicentric CE mark clinical investigation analyzing safety and effectiveness of the RefluxStop ${ }^{\mathrm{Tm}}$ device to treat GERD, 50 subjects with chronic GERD were included. Subjects were operated using a standardized surgical technique in the period between December 2016 and September 2017 and followed up for 1 year with the CE mark obtained based on 6month follow-up. Gastroscopy, contrast swallow x-ray, Questionnaires including GERD-HRQL [24] and foregut symptom [25] as well as 24-h pH monitoring were performed at Baseline and at 6 months after surgery. The GERD-HRQL score is used as a screening tool at the 1year follow-up to determine whether further investigations are necessary, including: additional contrast swallow x-ray and 24-h pH monitoring as well as gastroscopy and manometry (Fig. 2).

An independent Data Monitoring Committee (DMC) evaluated the safety data and selected efficacy data. The DMC could have recommended increasing the sample size if they found that this was motivated.
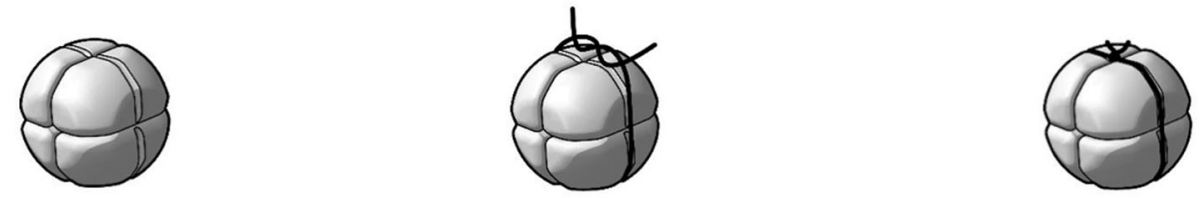

Fig. 1 RefluxStop ${ }^{T M}$ implant. RefluxStop ${ }^{T M}$ implant - illustration without suture, followed by assembly and suture placement 


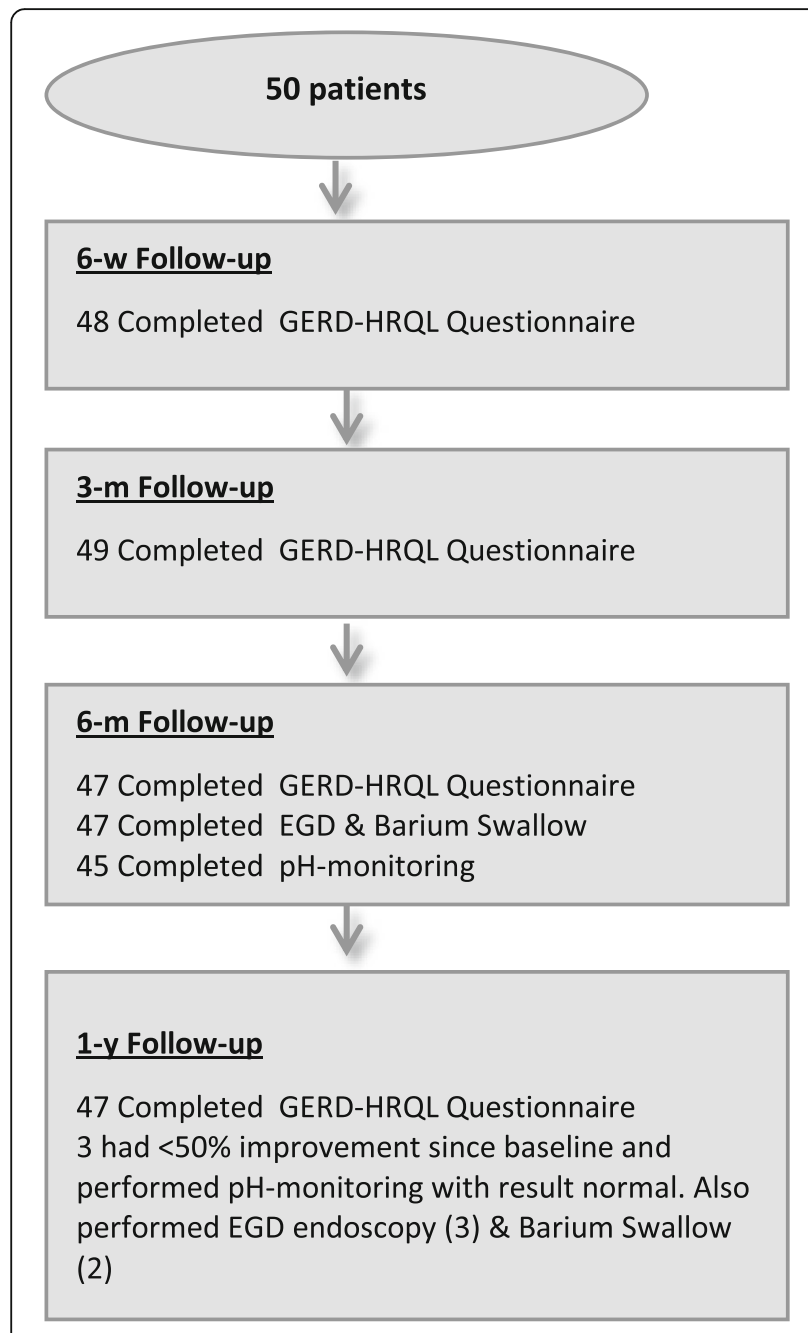

Fig. 2 Study Schedule of Assessments: Baseline to Year 1 visit

Primary safety objective was to assess the incidence of serious adverse device effects (SADEs) and procedurerelated serious adverse events (SAEs). Primary efficacy objective was to assess the percent reduction from baseline of GERD symptoms based on the GERD-HRQL total score.

Secondary safety objective was to assess the incidence of adverse device effects (ADEs) and procedure-related adverse events (AEs). Secondary efficacy objectives were to assess the reduction or normalization from baseline of the total acid $(\mathrm{pH}<4)$ exposure time on 24-h $\mathrm{pH}$ monitoring, to assess the reduction from baseline in the proportion of subjects using proton pump inhibitors (PPI) during the study, to assess the reduction from baseline of foregut questionnaire scores after the procedure and to assess the reduction from baseline of individual GERD symptoms based on the GERD-HRQL score.

Key study inclusion criteria were:
1. Subject's age $\geq 18$ years and $\leq 75$ years;

2. Subject has documented typical GERD symptoms present for $>6$ months which respond to PPIs as anti-GERD medication. Typical symptom of GERD is defined as heartburn, which is a burning epigastric or substernal pain;

3. Subject requires daily PPI anti GERD medication;

4. Subject has a 24-h pH monitoring proven GERD performed while off any anti-reflux medication or after discontinuation for at least 7 days prior to testing. Total distal esophageal $\mathrm{pH}$ must be $\leq 4$ for $\geq 4.5 \%$ of the time during a $24-\mathrm{h}$ monitoring.

Key study exclusion criteria were:

1. Subject has a history of gastroesophageal surgery, anti-reflux or bariatric procedure;

2. Presence of a para-esophageal hernia or sliding hernia of $>3 \mathrm{~cm}$ determined on endoscopy;

3. Presence of esophageal dysmotility disorder such as but not limited to scleroderma, achalasia,

Nutcracker esophagus;

4. Presence of an esophagitis grade $\mathrm{C}$ or $\mathrm{D}$ according to the Los Angeles classification;

5. Subject has a body mass index (BMI) $>35 \mathrm{~kg} / \mathrm{m}^{2}$;

The study was carried out in accordance with the Declaration of Helsinki, and the Regional Ethics Committee approved the study protocol.

\section{Study statistical analysis Statistical methods}

A sample size calculation was performed for this study where 45 subjects (50 including the drop-out rate) were determined to be sufficient. Continuous data is summarized using descriptive statistics and categorical data is presented using absolute frequency and percentage. The following analysis sets were considered in the statistical analysis:

- The full analysis set (FAS): in accordance with the intention-to-treat (ITT) principle, all subjects who received the device implant.

- The per-protocol (PP) analysis set: all subjects from FAS without any major protocol violations.

- The safety analysis set: all subjects who received the device implant.

- The primary efficacy variable was analyzed both in the FAS and in the PP analysis set. The secondary efficacy variables were analyzed in the FAS only. When timely results as per protocol were not available, subsequent results were used.

For all other variables, the safety analysis set was used. 


\section{Safety assessments}

All AE summaries described below are presented for each of the following groups of AEs: All reported AEs, ADEs, AEs related to surgery and AEs not defined as ADEs or surgery related AEs (i.e. not covered in 2 and 3 above). Summaries include: the number and percentage of subjects who reported at least $1 \mathrm{AE}$ and the number of events reported by seriousness, severity and outcome, and by system organ class (SOC) and preferred terms (PTs). Also, number of AEs leading to death were summarized.

Procedure-related complications (intra-operative complications or device related implanting difficulties, perioperative bleeding [>500 mL], peri-operative perforation, wound infection, pulmonary complications), length of the subjects' hospital stay, and weight are summarized descriptively. In addition, weight is also summarized as change from baseline.

\section{Efficacy assessments}

For the primary efficacy endpoint, the percent reduction from baseline of GERD symptoms based on the GERDHRQL total score (questions 1-10) is summarized using descriptive statistics, including $95 \% \mathrm{CI}$ of the mean percent reduction. Number of subjects with a worsening from baseline is summarized. Total score values for each subject and visit are also presented. The data is also presented as the number of subjects obtaining at least a $50 \%$ improvement of the baseline figures. The aim of this analysis was to show that the lower limit of the CI exceeded $60 \%$. Comparisons between values at baseline versus post-procedure follow-up were performed using a paired t-test at the 0.5 significance level. Month 6 is considered the primary efficacy endpoint. All secondary efficacy endpoints are presented descriptively, as appropriate.

\section{RefuxStop ${ }^{\mathrm{TM}}$ operating procedure}

All steps to treat hiatal hernia $(\mathrm{HH})$, if present, should be followed:

1. Hernia repair consisting of complete reduction of $\mathrm{HH}$, and sac excision if present

2. Extensive mediastinal esophageal dissection with vagal preservation

3. Gastroesophageal junction fat pad dissection to expose the angle of His

4. Intraoperative evaluation of esophageal length (with no traction)

5. About $1 \mathrm{~cm}$ additional dissection with small $1 \mathrm{~cm}$ down traction on esophagus

6. Tension-free crural repair

7. Left side adhering fundus to esophagus all the way up to the diaphragm (using the above mentioned small $1 \mathrm{~cm}$ down traction on esophagus)
8. GERD management with RefluxStop ${ }^{\mathrm{m}}$ as an antireflux procedure including placing RefluxStop ${ }^{\text {Tx }}$ in a pouch outside the top part of the fundus close to esophagus

Few issues need to be emphasized. For the new treatment principle to work the device needs to be placed high-up, clearly above the upper edge of the LES. To achieve such result; firstly, the dissection around esophagus in mediastinum needs to be more extensive and as high up as possible. Secondly, the adherence of the stomach fundus wall to the esophagus, which builds the platform for the device, should also be placed as high up as possible and include the esophagus on the subjects left side only.

The left lateral part of the esophagus should be attached to the stomach fundus wall. If the fundus is on the larger side, three parallel continuous non-resorbable sutures could be used, and when fundus is on the smaller side, two continuous sutures could be sutured in a Y-shape with short tail with one additional single suture in between the top of the Y (Fig. 3). The latter single suture could be replaced if sutures with hooks are used (for example non-resorbable V-Loc) with the top of the Y-shaped sutures angling slightly downwards and inwards towards each other to further stabilize both the sutures and the top part of the adherence between the fundus and the esophagus. We advocate one should avoid fat in the suture line attachments as much as possible and avoid suturing with too superficial sutures in the esophagus.

The placement of the device is performed using a special instrument that also compresses the device before introducing it. The device is then placed high-up on the outside of the stomach fundus wall and invaginated/covered by stomach tissue in a pouch to keep it in place, performed when holding the device with the instrument to ensure its high-up placement (Fig. 4).

Due to the importance of positioning the device highup and to ensure quality control of the procedure, the device shall be categorized using the following scale: 1 Optimal, top of the device placed $>1$ time the device size above the upper edge of the LES; 2 Acceptable, top of the device placed 0.5-1 time the device size above the upper edge of the LES; and 3 Failure Risk, top of the device placed 0-0.5 time the device size above the upper edge of the LES, which has a high risk of failure in the mid- to long-term; and in addition 4 Unacceptable, when the position of the RefluxStop ${ }^{\text {tw }}$ device is fully below the upper edge of the LES, in which case the device cannot function properly (likely leading to failure immediately or in the short- to mid-term) (Fig. 5).

It is also of utmost importance to place the device close to the esophagus for the device to function 


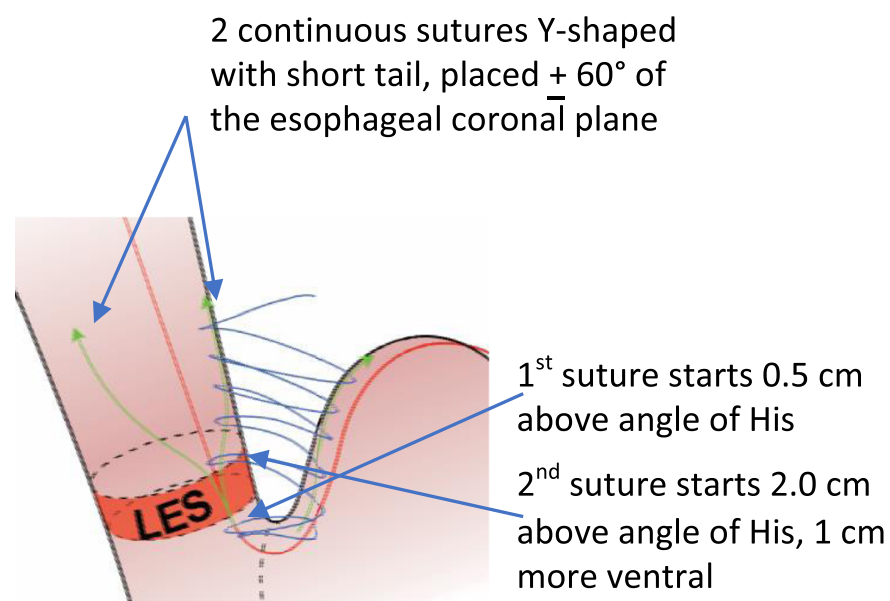

Approximetely $2.5 \mathrm{~cm}$ or $120^{\circ}$ of esophagus inbetween the sutures on top of the sutured $\mathrm{Y}$ with short tail

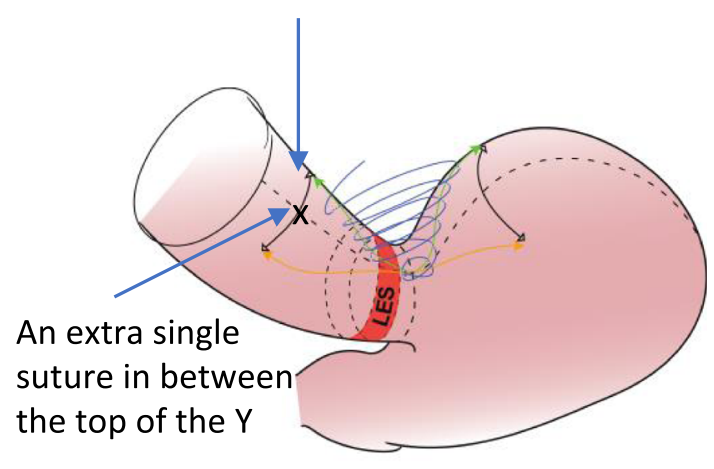

Fig. 3 Simplified stomach to esophagus Y-shaped suturing for smaller sized fundus

properly. Therefore, each height category should also be classified by Close $(\mathrm{C})$, a placement of $<1.5 \mathrm{~cm}$ from the esophagus where primarily just the thickness of the doubled stomach wall is placed between the device and the esophagus, or Distant (D), a placement further than 1.5 $\mathrm{cm}$ away from the esophagus (Fig. 5).

\section{Results}

\section{Study subjects}

The 50 subjects had the procedure performed at four different sites and have been followed for 1 year. Device implantation was attempted and performed in all subjects. Three subjects had discontinued the study by 1 year:

- 2 subjects with successful treatment results discontinued the study at 3 months and 6 months, respectively, with the following results at the time of discontinuation:
- No Dysphagia, no Odynophagia, no Regular Daily PPI consumption, no Regurgitation and subjects Satisfied

- 1 subject had the device placed too low at surgery refused re-surgery and discontinued at 6-months with the following results at the time of discontinuation:

- Moderate Dysphagia, no Odynophagia, no Regular Daily PPI consumption, minimal Regurgitation and subject Dissatisfied

\section{Baseline characteristics}

Subject mean age was: 51.5 (SD 11.8) years. Of the 50 subjects, $28(56 \%)$ were men and $22(44 \%)$ women. PPI consumption was halted for all subjects at least 1 week prior to the baseline visit. 24-h $\mathrm{pH}$ monitoring (percentage of overall time $\mathrm{pH}<4$ ) at baseline showed acid reflux in all subjects; mean result $16.35 \%$. The mean score for the GERD-HRQL questionnaire (questions 1-10) 1
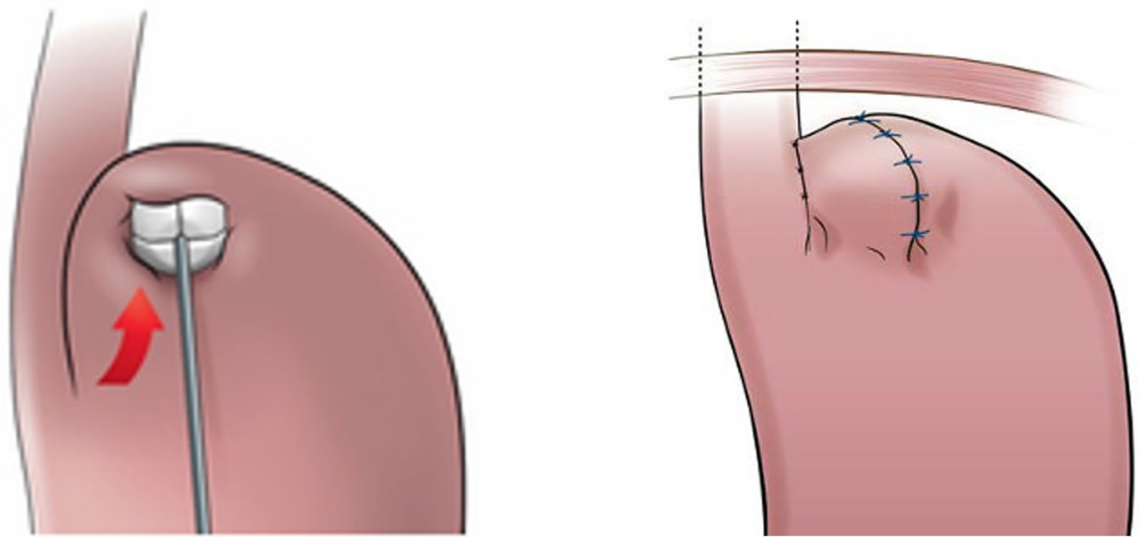

Fig. 4 The device is positioned using the RefluxStop ${ }^{\mathrm{TM}}$ deployment tool 


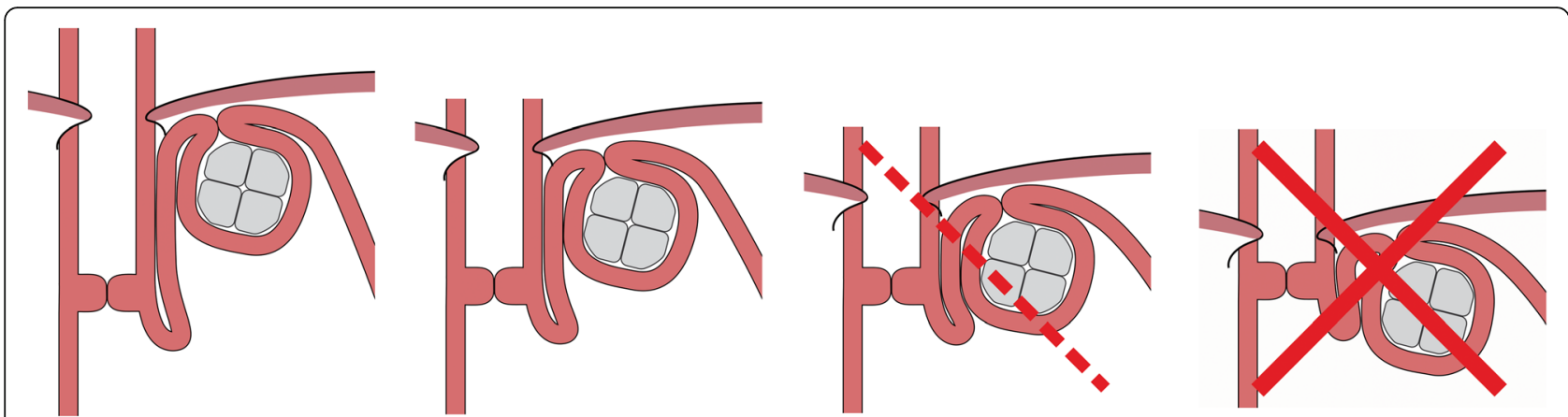

Fig. 5 RefluxStop ${ }^{\mathrm{Tm}}$ Height Categories. The categories of the device placement with a high-up placement important for a successful result. Optimal (1), top of the device placed $>1$ time the device size above the upper edge of the LES, Acceptable (2), top of the device placed $0.5-1$ time the device size above the upper edge of the LES, and Failure Risk (3), top of the device placed 0-0.5 time the device size above the upper edge of the LES, which has a high risk of failure in the mid- to long-term, and in addition Unacceptable (4), when the position of the RefluxStop ${ }^{\mathrm{TM}}$ device is fully below the upper edge of the LES in which case the device cannot function properly, (likely leading to failure immediately or in the short- to mid-term)

week off PPI at baseline was 28.8 (SD 7.3). Before surgery, all subjects took PPIs. At baseline endoscopy, 13 subjects (26.0\%) had Grade A esophagitis and 9 subjects (18.0\%) had Grade B esophagitis. At baseline, 26 subjects reported severe regurgitation in the foregut questionnaire. Forty-five of 50 subjects were Dissatisfied, 4 subjects Neutral and 1 subject Satisfied at baseline. The median score for severity of heartburn (GERD-HRQL question 1) was 4.0 at baseline (range 0 to 5). When assessed by the GERD-HRQL questionnaire at baseline, 15 subjects had difficulty swallowing (dysphagia) and 13 subjects had pain at swallowing (odynophagia). The median score for bloating or a gassy feeling was 4.0 (range 0 to 5 ) at baseline.

\section{Safety parameters}

No serious adverse events related to the RefluxStop ${ }^{\text {tin }}$ device were reported during the 6-month and 1-year follow-up periods. No deaths, no device deficiencies and no device explantations were reported/performed during this period.

The following presentation of the adverse events has been divided into events related to the device, anti-reflux surgery or surgical procedure in general (Table 1). Further division of events with respect to seriousness aims to facilitate an overview of the safety profile of the device.

\section{Serious adverse events related to surgery - none device related}

Six serious adverse events (SAEs) were reported for 4 subjects at 6 months. All but one of the events described above were resolved at the time of the 6-months analysis cut-off date. The subject diagnosed with an abdominal wall hernia unrelated to surgery may eventually undergo surgical repair.
Two of these adverse events above were more serious, directly correlated to surgery in general belonging to the two most common adverse events in surgery, namely one infection and one bleeding. The bleeding lead to a second look. It probably originated from the divided short gastric vessel, was self-limiting and clot evacuation and drainage was performed. The infection included both mediastinal abscess and empyema, probably due to the infected mediastinal haematoma. However, due to a well-performed invagination of the device the infection did not spread to the pouch with the device, and the subject healed completely with an excellent treatment result.

One serious adverse event (SAE) was reported in 1 subject between the 6-month and 1-year visit due to the release of fundoplication sutures. Reoperation was performed, and the subject had a successful 1-year follow-up visit. This confirms the treatment principle and reinforces the importance of positioning the device high up.

\section{Complications and side effects related to the procedure}

Dysphagia and odynophagia Two important complications related to anti-reflux surgery are dysphagia and odynophagia [1-3, 20]. Fifteen subjects had dysphagia at baseline, whereof in 11 completely resolved and 4 subjects continued to have reduced swallowing problems at the 6-month visit (GERD-HRQL score above 1) (Table 2). At the 1-year visit, 2 subjects reported minimal dysphagia. No new cases of dysphagia were recorded at either visit.

Thirteen subjects had pain at swallowing before surgery and none of the subjects reported pain at swallowing at 
Table 1 Adverse Events related to the device, anti-reflux surgery or surgery in general

\begin{tabular}{|c|c|c|c|}
\hline $\begin{array}{l}\text { Adverse Events during surgery and in the postoperative course up } \\
\text { to } 6 \text { months }\end{array}$ & $\begin{array}{l}\text { Number of subjects } \\
\mathrm{n}(\%)\end{array}$ & $\begin{array}{l}\text { Number of events } \\
\mathrm{n}\end{array}$ & $\begin{array}{l}\text { Full recovery with } \\
\text { intact treatment effect }\end{array}$ \\
\hline Number of Adverse Events $(n=50)$ & $8(16 \%)$ & 11 & YES \\
\hline Device related adverse events (SADE or ADE) & 0 & 0 & \\
\hline Surgical non-device related serious adverse events (SAE) & $4(8 \%)$ & 6 & YES \\
\hline \multicolumn{4}{|l|}{ Severe } \\
\hline Mediastinal abscess, empyema and abdominal abscess & $1^{a}$ & 3 & YES \\
\hline Intra-abdominal haemorrhage & 1 & 1 & YES \\
\hline \multicolumn{4}{|l|}{ Moderate } \\
\hline Pleuritis & 1 & 1 & YES \\
\hline \multicolumn{4}{|l|}{ Mild } \\
\hline Removal of foreign body (part of a needle from the abdominal wall) & 1 & 1 & YES \\
\hline Surgical non-device related non-serious adverse events (AE) & $4(8 \%)$ & 5 & YES \\
\hline \multicolumn{4}{|l|}{ Moderate } \\
\hline Abdominal pain and incisional hernia & $1^{\mathrm{b}}$ & 2 & YES \\
\hline \multicolumn{4}{|l|}{ Mild } \\
\hline Accidental intra-operative instrumental hepatic lesion (small) & 1 & 1 & YES \\
\hline Post-op delayed gastro-intestinal paralysis (one day) & 1 & 1 & YES \\
\hline Procedural pneumothorax & 1 & 1 & YES \\
\hline Adverse Events between 6 months and 1 year ${ }^{c}$ & $1(2 \%)$ & 1 & YES \\
\hline Device related adverse events (SADE or ADE) & 0 & 0 & \\
\hline Surgical non-device related serious adverse events (SAE) & $1(2 \%)$ & 1 & YES \\
\hline \multicolumn{4}{|l|}{ Moderate } \\
\hline Release of fundoplication sutures - successfully re-sutured & 1 & 1 & YES \\
\hline
\end{tabular}

The analysis is based on 50 subjects, i.e. all subjects included in the safety analysis set

a The events occurred in the same subject (caused by an infection unrelated to the device, which was unaffected in its enclosed pouch)

${ }^{\mathrm{b}}$ The events occurred in the same subject (a small hernia in the abdominal wall)

c Four AEs of gastritis occurred in 4 subjects all with endoscopy verified gastritis and lack of esophagitis, all resolved

Table 2 Dysphagia and Odynophagia at Baseline, 6-months and 1-year post-operation

\begin{tabular}{|c|c|c|c|c|c|c|c|c|}
\hline \multirow{2}{*}{$\begin{array}{l}\text { Dysphagia } \\
\text { None 0-1 }\end{array}$} & \multicolumn{2}{|c|}{ Baseline $(n=50)$} & \multicolumn{2}{|c|}{ 6-months $(n=47)$} & \multirow{2}{*}{$\begin{array}{l}\text { 6-m } \\
p \text {-value }\end{array}$} & \multicolumn{2}{|c|}{1 -year $(n=47)$} & \multirow{2}{*}{$\begin{array}{l}1 \text {-yr } \\
p \text {-value }\end{array}$} \\
\hline & 35 & $70 \%$ & 43 & $91 \%$ & & 45 & $96 \%$ & \\
\hline Minimal 2 & 4 & $30 \%$ & 3 & $9 \%$ & $<0.001$ & 2 & $4 \%$ & $<0.001$ \\
\hline Moderate 3 & \multicolumn{2}{|l|}{6} & \multicolumn{2}{|l|}{1} & & \multicolumn{2}{|l|}{0} & \\
\hline Severe 4-5 & \multicolumn{2}{|l|}{5} & \multicolumn{3}{|l|}{0} & \multicolumn{2}{|l|}{0} & \\
\hline Odynophagia & \multicolumn{2}{|c|}{ Baseline $(n=50)$} & \multicolumn{2}{|c|}{ 6-months $(n=47)$} & $\begin{array}{l}\text { 6-m } \\
p \text {-value }\end{array}$ & \multicolumn{2}{|c|}{ 1-year $(n=47)$} & $\begin{array}{l}1 \text {-yr } \\
p \text {-value }\end{array}$ \\
\hline None 0-1 & 37 & $74 \%$ & 47 & $100 \%$ & & 46 & $98 \%$ & \\
\hline Minimal 2 & 5 & $26 \%$ & 0 & $0 \%$ & $<0.001$ & 1 & $2 \%$ & \\
\hline Moderate 3 & 3 & & 0 & & & 0 & & $<0.001$ \\
\hline Severe 4-5 & 5 & & 0 & & & 0 & & \\
\hline
\end{tabular}




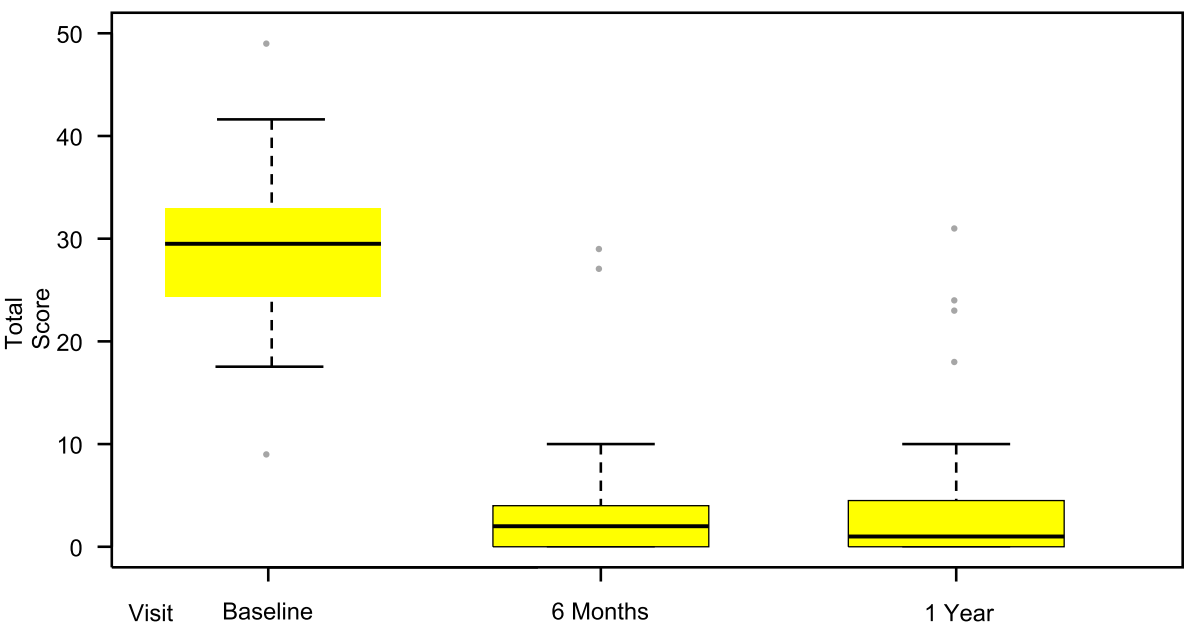

Fig. 6 Total GERD-HRQL score at Baseline, 6 months and 1 year post-operation. Total GERD-HRQL score including all 10 items before and 6 months after RefluxStop ${ }^{\mathrm{TM}}$ surgery, showing significant change from baseline to 6 months and 1-year $(p<0.001)$. Three subjects had less than $50 \%$ improvement of the score at 1-year, whereof two subjects due to reasons other than GERD and 1 subject due to a too low positioning of the device thereby prohibiting its function

the 6-month visit, while at the 1-year visit, one subject reported pain at swallowing at the same minimal level as at baseline. No new cases of odynophagia were recorded at the 6-month or 1-year visits (Table 2).

\section{Efficacy parameters}

\section{Reduction from baseline of GERD symptoms based on the GERD-HRQL score}

The mean total GERD-HRQL score at baseline was 28.8 (SD 7.3), $n=50$. At 6 months $(n=47)$, the score had decreased to 3.4 (SD 6.0) $(p<0.001)$, reflecting an improvement in GERD symptoms of $88 \%$. One subject discontinued the study with successful 3-month score (Fig. 6 and Table 3).

Forty-five out of 47 subjects had at least $50 \%$ improvement of the GERD-HRQL total score from baseline. Two subjects are defined as a failure not having a 50\% improvement in the GERD-HRQL score. Both these

Table 3 GERD-HRQL total score at 6 months and 1 year postoperation

\begin{tabular}{lll}
\hline Full analysis set & $\begin{array}{l}\text { Percent change Baseline } \\
\text { to 6 M }\end{array}$ & $\begin{array}{l}\text { Percent change Baseline } \\
\text { to } 1 \mathrm{Y}\end{array}$ \\
\hline $\mathrm{n}$ & 47 & 47 \\
Mean (SD) & $-89.0(17.9)$ & $-86.2^{\mathrm{a}}(24.5)$ \\
Median & -94.6 & -95.2 \\
Min, Max & $-100.0,-15.6$ & $-100.0,9.1$ \\
$p$-value & $<0.001$ & $<0.001$ \\
$95 \% \mathrm{Cl}$ & $(-94.3,-83.7 \%)$ & $(-93.4,-79.0 \%)$ \\
\hline
\end{tabular}

ancludes 2 subjects with $<50 \%$ improvement for reasons other than GERD and one with the device positioned improperly in a too low position subjects had the device positioned too low, prohibiting the device to function as intended. One subject had the device placed too low at surgery and refused re-surgery (representing proof of concept since an incorrectly placed device does not treat the subject). In the second subject, the suture line between the stomach and esophagus failed which is believed to be due to fat in the suture line attachment. The subject was well treated during the 3-month follow-up and was recorded as a failure after 6 months because the fundus with the intact invaginated device had slipped down due to the stomach-to-fundus suture line failing, resulting in the device being positioned too low, hindering its function. The subject was reoperated after 8 months with resuturing and was immediately treated with optimal results (GERD-HRQL total score zero points) supporting once again that the new treatment principle works.

At the 1-year follow-up visit $(n=47),>50 \%$ improvement in GERD symptoms compared to baseline was reported in 44 subjects. Two subjects with $<50 \%$ improvement were shown to not have GERD and one subject had the device positioned improperly too low. The average score improvement when deducting the two subjects with failed results for reasons other than GERD was $89 \%$ and average score was 3.2. Full results in Fig. 6 and Table 3.

\section{4-h pH monitoring}

$\mathrm{pH}$ testing at the 6 months study visit was completed by 45 subjects. The esophageal $\mathrm{pH}$ was monitored using Bravo Capsules during a 24-h period at baseline and again at 6 months post-implantation (Fig. 7 and Table 4). The results show a mean reduction from baseline of percentage of 

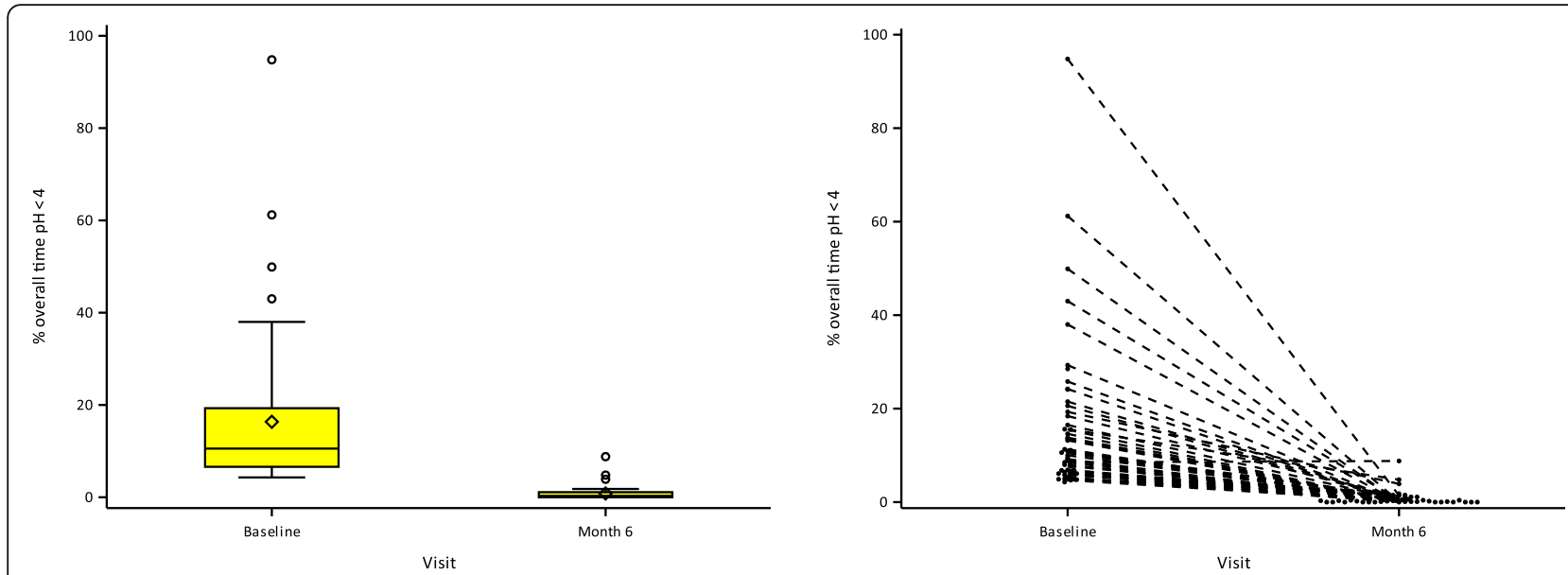

Fig. 7 24-h pH monitoring at Baseline and 6 months post-operation. 24-h pH monitoring mean reduction from baseline of 16.35 to $0.80 \%$ at $6-$ month visit. Normal $24-\mathrm{h}$ pH results in $98 \%$ of subjects

overall time with $\mathrm{pH}<4$ from 16.35 to $0.80 \%$ at the 6month visit $(p<0.001)$ reflecting a $95 \%$ improvement of the mean value. Normal 24-h pH results in $98 \%$ of subjects.

Two of the subjects refused the burdensome $\mathrm{pH}$ monitoring test whereof one had a successful GERDHRQL questionnaire score and one subject had the device positioned too low at surgery, prohibiting the function of the device. The latter subject refused reoperation and the $\mathrm{pH}$ monitoring test, but notable that this subject did not take any PPIs.

\section{PPI medication}

PPI consumption/reuse is often used as an indicator for success in reflux surgery. Before surgery all 50 subjects were taking PPI drugs. Six months after implantation no subject (0\%), $n=47$, took PPI medication. At the 1 -year follow-up visit, 1 subject (2.1\%), $n=47$, who had the device positioned too low, took regular daily PPIs (Table 5).

Table 4 24-h pH monitoring at Baseline and 6 months postoperation

\begin{tabular}{llll}
\hline $\begin{array}{l}\text { \% overall } \\
\text { time } \mathrm{pH}<4\end{array}$ & Baseline & 6 Months & $\begin{array}{l}\text { Change Baseline to } \\
6 \text { Months }\end{array}$ \\
\hline $\mathrm{n}$ & 50 & 45 & 45 \\
Mean (SD) & $16.35 \%(16.60)$ & $0.80 \%(1.56)$ & $-16.0(17.46)$ \\
Median & $10.55 \%$ & $0.30 \%$ & -10.05 \\
$\begin{array}{l}\text { Min, Max } \\
p \text {-value* }\end{array}$ & $4.3,94.8$ & $0.0,8.8$ & $-93.4,0.3$ \\
\hline
\end{tabular}

* Paired t-test of the mean change

Two of the subjects refused the burdensome $\mathrm{pH}$-monitoring test whereof one had a successful GERD-HRQL questionnaire score and one subject had a to too low position of the device already at surgery, prohibiting the function of the device. This later subject refused the $\mathrm{pH}$ monitoring test and re-surgery

\section{Gas bloating}

Gas bloating often occurs in anti-reflux surgery $[1-3,17]$ and in the investigation the question about gas bloating in the GERD-HRQL (score above 2) was present in $84.0 \%$ of the subjects at baseline and in $19.1 \%$ of the subjects at 1 year. Gas bloating at 1 year compared to baseline: disappeared in 30 subjects; improved in 7 subjects; remained unchanged in 2 subjects; and no subject had their gas bloating symptoms worsen (Table 6).

\section{Regurgitation}

Daily regurgitation is common in acid reflux subjects [3] and occurred in 44 subjects (88\%) at baseline in the investigation, whereof 33 subjects had moderate to severe regurgitation. At 1-year follow-up 46 out of 47 evaluable subjects $(97.8 \%)$ operated with RefluxStop ${ }^{\mathrm{Tm}}$ had none or minimal occasional episodes of regurgitation (Table 7).

\section{Subject satisfaction}

Forty-five subjects at baseline indicated that they were dissatisfied with their present condition whereas at

Table 5 PPI consumption before surgery and at follow up visits

\begin{tabular}{lllll}
\hline & $\begin{array}{l}\text { Number of } \\
\text { subjects }(\mathrm{n})\end{array}$ & $\begin{array}{l}\text { Total } \\
\mathrm{n}(\%)\end{array}$ & $\begin{array}{l}\text { PPI use } \\
\text { related to } \\
\text { acid reflux }\end{array}$ & $\begin{array}{l}\text { PPI use related } \\
\text { to malfunction } \\
\text { of the treatment } \\
\text { principle of } \\
\text { RefluxStop }\end{array}$ \\
\hline $\begin{array}{llll}\text { RM } \\
\text { Regular daily PPI consumption }\end{array}$ & $50(100 \%)$ & $50(100 \%)$ & $50(100 \%)$ \\
Baseline & 50 & $0(0.0 \%)$ & 0 & 0 \\
1 Mear & 47 & $1(2.1 \%)$ & $1^{\text {a }}$ & 0 \\
\hline
\end{tabular}

${ }^{\mathrm{a}}$ One (1) subject with the device positioned too low, prohibiting the function of the device 
Table 6 Gas bloating including change between Baseline and 1-year

\begin{tabular}{|c|c|c|c|c|c|c|c|c|c|}
\hline \multirow{2}{*}{$\begin{array}{l}\text { Gas bloating } \\
\text { None } 0-2\end{array}$} & \multicolumn{2}{|c|}{ Baseline $(n=50)$} & \multicolumn{2}{|c|}{$\begin{array}{l}\text { 6-months } \\
(n=47)\end{array}$} & \multirow[t]{2}{*}{$\begin{array}{l}\text { 6-m } \\
p \text {-value }\end{array}$} & \multicolumn{2}{|c|}{ 1-year $(n=47)$} & \multirow[t]{2}{*}{$\begin{array}{l}1 \text {-yr } \\
p \text {-value }\end{array}$} & \multirow{2}{*}{$\begin{array}{l}\text { Gas bloating change between } \\
\text { Baseline and 1-year (subjects) } \\
\text { Disappeared in } 30\end{array}$} \\
\hline & 8 & $16 \%$ & 37 & $79 \%$ & & 38 & $81 \%$ & & \\
\hline Minimal 3 & 10 & $84 \%$ & 5 & $21 \%$ & $<0.0001$ & 5 & $19 \%$ & $<0.0001$ & Improved in 7 \\
\hline Moderate 4 & 18 & & 3 & & & 3 & & & Unchanged in 2 \\
\hline Severe 5 & 14 & & 2 & & & 0 & & & Worsened in 0 \\
\hline
\end{tabular}

follow-up 6 months after implantation 44 subjects (93.6\%) were satisfied, one subject was neutral $(2.1 \%)$ and two subjects dissatisfied $(4.3 \%)(n=47)$ (Table 8$)$. The two dissatisfied subjects were the same subjects as discussed previously with the device placed too low, hindering its function. Out of these, one subject was reoperated between 6 months and 1 year to improve the positioning of the device by placing it higher up and was thereafter immediately satisfied again (HRQL total score zero).

At the 1-year follow-up, one subject was still neutral (2.1\%) and one subject was dissatisfied due to the device being positioned too low. In addition, two subjects (4.3\%) were dissatisfied for reasons other than GERD: one subject had all tests performed with a normal outcome and one (1) subject had short-term gastritis and was satisfied at the subsequent follow-up visit and 2-year visit.

So far, no correctly operated subject has failed, which means that there is a $100 \%$ success rate for the treatment principle of RefluxStop ${ }^{\mathrm{Tm}}$, supporting a possible shift in acid reflux treatment.

\section{Summary of the safety endpoint}

No device related serious or non-serious adverse events occurred during the study. The surgery related serious adverse events were all resolved with satisfactory results.

\section{Summary of the main efficacy endpoint GERD-HRQL}

- $86 \%$ score reduction from baseline at 1 -year follow up visit

- $95 \%$ confidence interval: $94.1-79.4 \%$

- Lower limit of confidence interval was above $60 \%$

Table 7 Daily regurgitation before surgery and at 1-year follow up visit

\begin{tabular}{llllll}
\hline $\begin{array}{l}\text { Daily regular } \\
\text { Regurgitation }\end{array}$ & Baseline $(\mathrm{n}=50)$ & 1-year $(\mathrm{n}=47)$ & $\begin{array}{l}\text { 1-yr } \\
p \text {-value }\end{array}$ \\
\hline None $<$ 1/day & 6 & $12 \%$ & 43 & $91 \%$ & \\
Minimal 1-2/day & 11 & $88 \%$ & 3 & $9 \%$ & $<0.0001$ \\
Moderate 3-4/day & 16 & & 1 & & \\
Severe 5 or more per day & 17 & & 0 & & \\
\hline
\end{tabular}

- Percent of subjects with at least 50\% improvement: $93.6 \%$

- 3 subjects had <50\% improvement, whereof:

- 2 were shown not to have GERD

- 1 due to too low position of the device thereby prohibiting its function

24-h pH Monitoring:

- $98 \%$ of subjects had normal 24-h pH monitoring (percentage of total time) at 6-months

- $95 \%$ improvement at 6-months on mean value

- $16.35 \%$ at Baseline reduced to $0.80 \%$ at 6 -months $(p<0.001)$

\section{Discussion}

The new device RefluxStop ${ }^{\text {тM }}$ uses a new dynamic acid reflux treatment principle, which does not compress the food passageway. The 1 -year results indicate that the new principle for treating acid reflux is successful for all correctly operated subjects.

That a dynamic treatment for acid reflux, which avoids compressing the food passageway, would reduce complications such as dysphagia and odynophagia, as well as gas bloating, in relation to standard of care methods would be expected and is supported by the clinical trial results. No new diagnosed case of dysphagia was detected. In addition, dysphagia scores improved significantly at the 1-year follow-up visit compared to baseline. Furthermore, there were no device deficiencies and no complications related to the device itself.

Table 8 Subject satisfaction before surgery and at follow-up visits

\begin{tabular}{|c|c|c|c|}
\hline Subject Satisfaction & $\begin{array}{l}\text { Baseline } \\
(n=50)\end{array}$ & $\begin{array}{l}\text { 6-months } \\
(\mathrm{n}=47)\end{array}$ & $\begin{array}{l}\text { 1-year } \\
(n=47)\end{array}$ \\
\hline Satisfied & 1 & 44 & 43 \\
\hline Neutral & 4 & 1 & 1 \\
\hline Dissatisfied & 45 & 2 & 1 \\
\hline Dissatisfied - not due to GERD & 0 & 0 & $2^{a}$ \\
\hline \multicolumn{4}{|c|}{$\begin{array}{l}\text { Two of the dissatisfied subjects were dissatisfied for reasons other } \\
\text { than GERD: } \\
\text { - One (1) subject had normal gastroscopy, 24-h pH monitoring and contrast } \\
\text { swallow x-ray; and } \\
\text { - One (1) subject had gastritis and regained satisfaction after a short-term non- } \\
\text { PPI treatment, still satisfied at the 2-year follow-up }\end{array}$} \\
\hline
\end{tabular}


Gas bloating is a common finding after fundoplication [26]. Interestingly, the prevalence of gas bloating is surprisingly reduced after RefluxStop ${ }^{\mathrm{TM}}$ treatment. One out of many reasons causing gas bloating could be inability to turn back swallowed air, either by belching or more likely due to unnoticeable air exchange over the LES. Avoiding compression of the esophagus allows swallowed air to be released up the esophagus thereby reducing gas bloating. Thus, these one-year results already indicate that this new dynamic acid reflux treatment principle is here to stay.

More surprisingly there are also indications that the treatment efficacy of the RefluxStop ${ }^{\mathrm{Tm}}$ procedure may be an improvement over the current standard procedures. RefluxStop $^{\text {TM }}$ is effective to treat GERD symptoms and significantly reduces esophageal acid exposure. The GERD-HRQL questionnaire results show $86 \%$ score reduction from baseline at 1-year follow up visit and 89\% score reduction when excluding the two subjects who had failed score result for reasons other than GERD. In addition, results obtained by $24-\mathrm{h} \mathrm{pH}$ monitoring showed $95 \%$ improvement at 6 -months on mean value down to an average $0.8 \%$ of total time $\mathrm{pH}<4$. No other reason for treatment failure than inaccurate too low position of the device has been identified so far.

The new RefluxStop ${ }^{\mathrm{Tm}}$ anti-reflux procedure has two important areas of consideration: Firstly, the device only works if it is placed clearly higher up than the upper edge of the lower esophageal sphincter. The platform for achieving such a result is to maximize the esophagus dissection all the way up in mediastinum (type B dissection) [27]. Then gastroesophageal junction fat pad dissection to expose the angle of His and an optimal reconstruction of the angle of His are mandatory, allowing a high-up placement of the device. Incorrect placement will result in treatment failure with no or limited reflux symptom relief, as suggested by the two subjects with the device placed too low, and correct placement of the device will result in a successfully treated subject. One subject had the device placed too low already at surgery, see two x-rays (Figs. 8 and 9). In addition, due to some expected adaption of tissue and degeneration of the thickness of the fundus top wall as a result of immobilization, the device positioning is a key factor for long-term success.

The second important consideration is the adherence of the stomach fundus wall to esophagus on the left lateral side, which builds the platform for the device and should be performed as high up as possible. The esophagus should be robustly attached to the stomach fundus wall. If the fundus is a bit larger in size, three parallel vertical continuous sutures could be used, and when the fundus is smaller, two continuous sutures could be sutured in a Y-shape with short tail and one additional single suture in between the top of the $\mathrm{Y}$ -

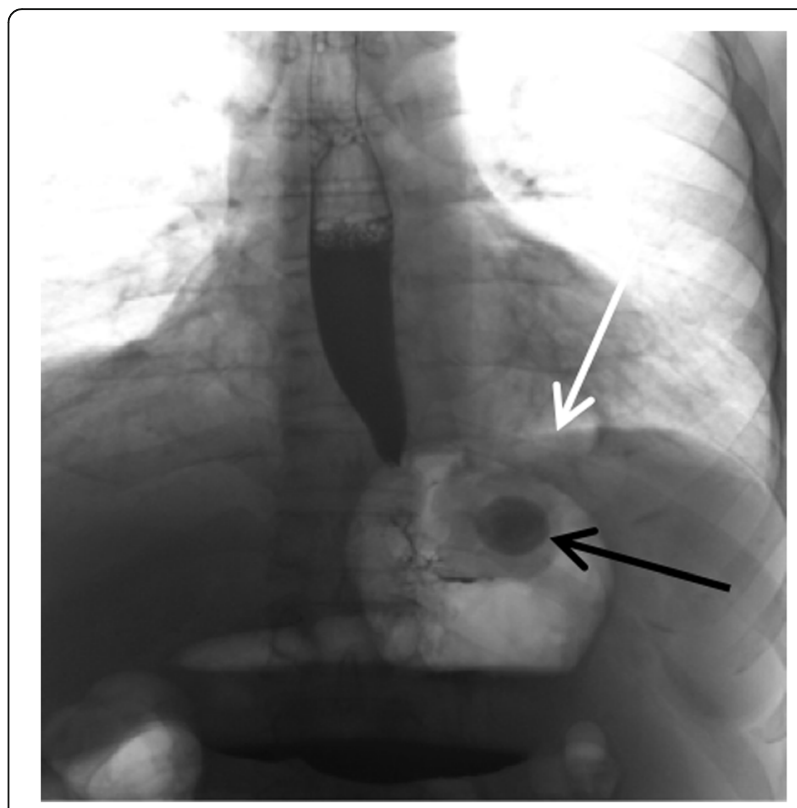

Fig. 8 The RefluxStop ${ }^{\text {TM }}$ device placed too low

shaped sutures. We advocate that one should avoid fat in the suture line attachments and avoid suturing with too superficial sutures in the esophagus.

The placement of the device with the specially designed instrument using compression of the device during introduction is a small part of the procedure. The instrument is used to hold the device in a high-up position when invaginated/covered completely by the stomach fundus wall.

Similar to existing anti-reflux surgery, the procedure as such can have complications of importance that require special care: bleeding and infection [28]. Infection could be a serious issue when placing an implant, however, the consequences of an infection are reduced if the device is completely covered/invaginated in the stomach wall by stomach-to-stomach sutures, thereby avoiding that the infection is spread to the device. Specific care is required to avoid gastric or esophagus injury/perforation. Because the esophagus is lacking a serosa that protects the muscle wall, sutures could cut through the muscular tissue if one is not careful enough.

We found the GERD-HRQL questionnaire to be a reliable screening tool to find subjects who need to perform 24-h pH monitoring. A failed questionnaire could occur for several reasons other than GERD, however, a successful questionnaire is very reliable and may only involve so-called silent acid reflux, subjects who are not included in the study from the beginning due to lack of symptoms. Most importantly, this group of subjects who have a failed questionnaire (symptoms of acid reflux) is more motivated to perform the often burdensome 24-h $\mathrm{pH}$ monitoring. 


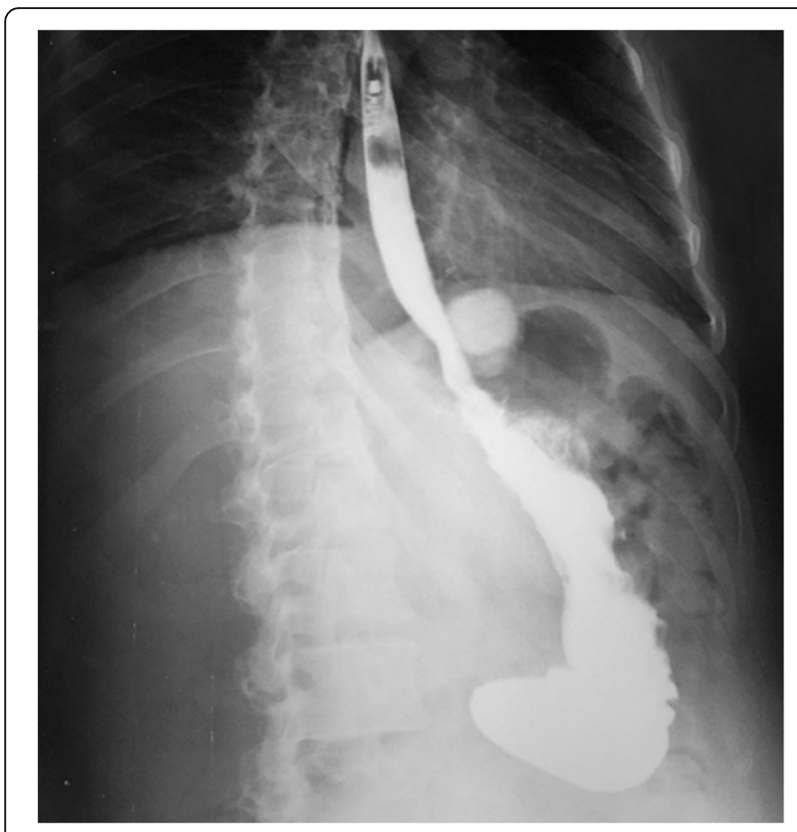

Fig. 9 A correctly placed RefluxStop ${ }^{\mathrm{TM}}$ device

A larger PMCF survey is currently being set up to further evaluate subjects long-term. The results so far indicate that RefluxStop ${ }^{\text {tw }}$ may become a breakthrough in acid reflux treatment.

\section{Conclusion}

The study results confirm that RefluxStop ${ }^{\mathrm{ma}}$ is safe and well tolerated with no complications related to the RefluxStop $^{\text {ti }}$ device itself (no SADE or ADE). Overall, data from the present study show that the complications caused by the compression of the food passageway i.e. dysphagia, odynophagia and gas bloating are not an issue with the RefluxStop ${ }^{\mathrm{Tm}}$ procedure. Both the GERD-HRQL score and 24- $\mathrm{h} \mathrm{pH}$ monitoring results indicate success for the new treatment principle in all correctly operated subjects. These 1-year results are very promising and RefluxStop $^{\text {Tu }}$ may cause a shift in acid reflux treatment, although further studies and follow-up are needed.

\footnotetext{
Abbreviations

GERD: Gastroesophageal reflux disease; HRQL: Health Related Quality of Life Questionnaire; CE-Mark: Conformité Européenne, a European certification mark; LES: Lower esophageal sphincter; PPI: Proton Pump Inhibitor; FAS: Full analysis set; PP: Per-protocol; ITT: Intention-to-treat; AE: Adverse events; SAE: Serious adverse events; SADE: Serious adverse device effects; ADE: Adverse device effects; DMC: Data Monitoring Committee; SOC: System organ class; PT: Preferred terms; Cl: Confidence interval; HH: Hiatal hernia; PMCF: Post-market clinical follow-up
}

\section{Acknowledgements}

Special thanks to Prof. Piet Pattyn and Dr. Elke Van Daele from Ghent University Hospital, Ghent, Belgium who provided initial contribution to the operating technique and study design.

Sub-Investigators of the Reflux Stop Clinical Investigation Study Group
Dragan Gunjić A ${ }^{\text {, }}$ Milan Veselinović ${ }^{A}$, Tamara Babič $^{\text {A }}$, Péter Lukovich ${ }^{\mathrm{B}}$, Timea Kakucs ${ }^{B}$, Sándor Kathy ${ }^{\mathrm{C}}$

A Department for Minimally Invasive Upper Digestive Surgery, University Hospital for Digestive Surgery - First Surgical Hospital, Clinical Center of Serbia; University of Belgrade, School of Medicine, Belgrade, Serbia.

B 1st Department of Surgery, Semmelweis University, Budapest, Hungary.

c University of Debrecen Kenézy Gyula Teaching Hospital, General Surgery

Department, Debrecen, Hungary.

\section{Authors' contributions}

$\mathrm{LH}$ was the lead investigator surgeon; $\mathrm{LH}, \mathrm{MB}, \mathrm{ZK}$ and $\mathrm{AA}$ recruited the subjects and collected the data; MB and PF contributed to the study design and operating technique and third party PCG PharmaConsulting Group AB (name changed to Link Medical) handled the data collecting system Viedoc and collected and analyzed the data; MB wrote the article with input from PF and LH and summarized the results supplied; LH, MB and PF contributed with intellectual content; All authors were asked about feedback on the final manuscript. The authors read and approved the final manuscript.

\section{Funding}

Funding was provided by Implantica, Vaduz, Liechtenstein, and Zug,

Switzerland, which financed the costs of the clinical investigation to achieve a CE mark for their new implantable device, RefluxStop ${ }^{\mathrm{Tm}}$.

\section{Availability of data and materials}

The datasets used and/or analyzed during the current study are available from the corresponding author on reasonable request.

\section{Ethics approval and consent to participate}

Informed written consent was obtained from all individual participants included in the study. The study was reviewed by:

Ethics Committee: Hungary

The Medical Research Council (MRC), Scientific and Research Ethics Committee (SREC) ${ }^{A}$, Alkotmány u. 25, H-1054 Budapest, Hungary.

${ }^{A}$ An independent Central Ethics Committee (CEC) responsible for the independent review and approval for all medical device trials in Hungary. Trial approval reference number: 048734/2016/OTIG, issued by the Hungary Regulatory Authority the National Institute of Pharmacy and Nutrition. Ethics Committee: Serbia.

Ethics Committee, Clinical Center of Serbia, 2 Pasterova Street, 110000 Belgrade, Serbia.

\section{Consent for publication}

All subjects have signed an Informed Consent Form in relation to their participation in the CE-mark clinical investigation RXI 001 of RefluxStop ${ }^{\mathrm{TM}}$, which provides consent for the publication of anonymized data.

\section{Competing interests}

$\mathrm{PF}$ is the inventor of the RefluxStop ${ }^{\mathrm{TM}}$ device, a specialist surgeon with focus on upper gastrointestinal surgery such as obesity and anti-reflux surgery. PF has been working for Implantica, the manufacturer of RefluxStop ${ }^{\mathrm{TM}}$ and the funder of the study. The other five authors ( $L H, M B, Z K$ and $A A)$ declare that they have no competing interests.

\section{Author details}

'Department for Minimally Invasive Upper Digestive Surgery, University Hospital for Digestive Surgery - First Surgical Hospital, Clinical Center of Serbia; University of Belgrade, School of Medicine, Belgrade, Serbia. ${ }^{2} 1 \mathrm{st}$ Department of Surgery, Semmelweis University, Budapest, Hungary. ${ }^{3}$ Surgical Department, Fejér County Szent György University Teaching Hospital, Székesfehérvár, Hungary. ${ }^{4}$ General Surgery Department, University of Debrecen Kenézy Gyula Teaching Hospital, Debrecen, Hungary. ${ }^{5}$ nventor of RefluxStop ${ }^{\mathrm{TM}}$, Seehof 4b, 6072 Sachseln, Switzerland.

Received: 26 February 2020 Accepted: 9 June 2020

Published online: 20 July 2020

\section{References}

1. Ackroyd R, Watson DI, Majeed AW, Troy G, Treacy PJ, Stoddard CJ. Randomized clinical trial of laparoscopic versus open fundoplication for gastro-oesophageal reflux disease. Br J Surg. 2004;91(8):975-82. 
2. Blomqvist A, Dalenback J, Hagedorn C, Lonroth H, Hyltander A, Lundell L. Impact of complete gastric fundus mobilization on outcome after laparoscopic total fundoplication. J Gastrointest Surg. 2000;4(5):493-500.

3. Booth MI, Stratford J, Jones L, Dehn TCB. Randomized clinical trial of laparoscopic total (Nissen) versus posterior partial (Toupet) fundoplication for gastro-oesophageal reflux disease based on preoperative oesophageal manometry. Br J Surg. 2008;95(1):57-63.

4. Broeders JA, Rijnhart-de Jong HG, Draaisma WA, Bredenoord AJ, Smout AJ, Gooszen HG. Ten-year outcome of laparoscopic and conventional nissen fundoplication: randomized clinical trial. Ann Surg. 2009;250(5):698-706.

5. Broeders JA, Broeders EA, Watson DI, Devitt PG, Holloway RH, Jamieson GG. Objective outcomes 14 years after laparoscopic anterior 180-degree partial versus Nissen fundoplication: results from a randomized trial. Ann Surg. 2013;258(2):233-9.

6. Cai W, Watson DI, Lally CJ, Devitt PG, Game PA, Jamieson GG. Ten-year clinical outcome of a prospective randomized clinical trial of laparoscopic Nissen versus anterior $180^{\circ}$ partial fundoplication. Br J Surg. 2008:95(12): $1501-5$.

7. Chew CR, Jamieson GG, Devitt PG, Watson DI. Prospective randomized trial of laparoscopic Nissen fundoplication with anterior versus posterior hiatal repair: late outcomes. World J Surg. 2011;35(9):2038-44.

8. Chrysos E, Tzortzinis A, Tsiaoussis J, Athanasakis H, Vasssilakis J, Xynos E. Prospective randomized trial comparing Nissen to Nissen-Rossetti technique for laparoscopic fundoplication. Am J Surg. 2001;182(3):215-21.

9. Chrysos E, Tsiaoussis J, Athanasakis E, Zoras O, Vassilakis JS, Xynos E. Laparoscopic vs open approach for Nissen fundoplication. A comparative study. Surg Endosc. 2002;16(12):1679-84.

10. Djerf P, Montgomery A, Hallerback B, Hakansson HO, Johnsson F. One- and ten-year outcome of laparoscopic anterior $120^{\circ}$ versus total fundoplication: a double-blind, randomized multicenter study. Surg Endosc. 2016;30(1):168-77.

11. Draaisma WA, Buskens E, Bais JE, Simmermacher RK, Rijnhart-de Jong HG, Broeders IA, et al. Randomized clinical trial and follow-up study of costeffectiveness of laparoscopic versus conventional Nissen fundoplication. Br J Surg. 2006;93(6):690-7 (a).

12. Granderath FA, Schweiger UM, Kamolz T, Asche KU, Pointner R. Laparoscopic Nissen fundoplication with prosthetic hiatal closure reduces postoperative intrathoracic wrap herniation: preliminary results of a prospective randomized functional and clinical study. Arch Surg. 2005; 140(1):40-8.

13. Guérin $\mathrm{E}$, Betroune $\mathrm{K}$, Closset J, Mehdi A, Lefebvre JC, Houben JJ, et al. Nissen versus Toupet fundoplication: results of a randomized and multicenter trial. Surg Endosc. 2007;21(11):1985-90.

14. Khan MA, Smythe A, Globe J, Stoddard CJ, Ackroyd R. Randomized controlled trial of laparoscopic Nissen versus Lind fundoplication for gastrooesophageal reflux disease. Scand J Gastroenterol. 2009;44(3):269-75.

15. Laine S, Rantala A, Gullichsen R, Ovaska J. Laparoscopic vs conventional Nissen fundoplication. A prospective randomized study. Surg Endosc. 1997; 11(5):441-4

16. Mardani J, Lundell L, Lonroth H, Dalenback J, Engstrom C. Ten-year results of a randomized clinical trial of laparoscopic total fundoplication with or without division of the short gastric vessels. Br J Surg. 2009;96(1):61-5.

17. Mickevičius $A$, Endzinas Z, Kiudelis $M$, Jonaitis L, Kupčinskas L, Pundzius J, et al. Influence of wrap length on the effectiveness of Nissen and Toupet fundoplications: 5-year results of prospective, randomized study. Surg Endosc. 2013:27(3):986-91.

18. Müller-Stich BP, Reiter MA, Mehrabi A, Wente MN, Fischer L, Koninger J, et al. No relevant difference in quality of life and functional outcome at 12 months' follow-up-a randomised controlled trial comparing robot-assisted versus conventional laparoscopic Nissen fundoplication. Langenbecks Arch Surg. 2009;394(3):441-6.

19. Nakadi IE, Melot C, Closset J, DeMoor V, Betroune K, Feron P, et al. Evaluation of da Vinci Nissen fundoplication clinical results and cost minimization. World J Surg. 2006;30(6):1050-4.

20. Nijjar RS, Watson DI, Jamieson GG, Archer S, Bessell JR, Booth M, et al. Fiveyear follow-up of a multicenter, double-blind randomized clinical trial of laparoscopic Nissen vs anterior 90 degrees partial fundoplication. Arch Surg. 2010;145(6):552-7.

21. Salminen P, Hurme S, Ovaska J. Fifteen-year outcome of laparoscopic and open Nissen fundoplication: a randomized clinical trial. Ann Thorac Surg. 2012:93(1):228-33.
22. Yang H, Watson DI, Lally CJ, Devitt PG, Game PA, Jamieson GG. Randomized trial of division versus nondivision of the short gastric vessels during laparoscopic Nissen fundoplication: 10-year outcomes. Ann Surg. 2008; 247(1):38-42.

23. Karolinska Institute. Literature review and analysis, Laparoscopic Nissen fundoplication; 2018. p. 1-50.

24. Velanovich V, Vallance SR, Gusz JR, Tapia FV, Harkabus MA. Quality of life scale for gastroesophageal reflux disease. J Am Coll Surg. 1996;183(3):217-24.

25. Konstantinos IM, Cassera MA, Kastenmeier AS, Dunst CM, Swanström LL. Postoperative dysphagia is not predictive of long-term failure after laparoscopic antireflux surgery. Surg Endosc. 2011. https://doi.org/10.1007/ s00464-011-1898-4.

26. Kinsey-Trotman SP, Devitt PG, Bright T, Thompson SK, Jamieson GG, Watson DI. Randomized trial of division versus nondivision of short gastric vessels during Nissen fundoplication: 20-year outcomes. Ann Surg. 2018;268(2):22832. https://doi.org/10.1097/SLA.0000000000002648.

27. O'Rourke RW, Khajanchee YS, Urbach DR, Lee NN, Lockhart B, Hansen PD, Swanstrom LL. Extended transmediastinal dissection: an alternative to gastroplasty for short esophagus. Arch Surg. 2003;138(7):735-40 PMID: 12860754.

28. Yadlapati R, Hungness ES, Pandolfino JE. Complications of Antireflux surgery Am J Gastroenterol. 2018;113(8):1137-47. https://doi.org/10.1038/s41395018-0115-7.

\section{Publisher's Note}

Springer Nature remains neutral with regard to jurisdictional claims in published maps and institutional affiliations.
Ready to submit your research? Choose BMC and benefit from:

- fast, convenient online submission

- thorough peer review by experienced researchers in your field

- rapid publication on acceptance

- support for research data, including large and complex data types

- gold Open Access which fosters wider collaboration and increased citations

- maximum visibility for your research: over $100 \mathrm{M}$ website views per year

At BMC, research is always in progress.

Learn more biomedcentral.com/submissions 